Progress in Flight Physics 5 (2013) 57-68

DOI: $10.1051 /$ eucass/201305057

(C) Owned by the authors, published by EDP Sciences, 2013

\title{
DIRECT NUMERICAL SIMULATIONS OF MACK-MODE DAMPING ON POROUS COATED CONES
}

\author{
H. Lüdeke and V. Wartemann \\ DLR Institute of Aerodynamics and Flow Technology \\ 7 Lilienthalplatz, Braunschweig 38108, Germany
}

The flow field over a 3 degree blunt cone is investigated with respect to a hypersonic stability analysis of the boundary-layer flow at Mach 6 with porous as well as smooth walls by comparing local direct numerical simulations (DNS) and linear stability theory (LST) data. The original boundary-layer profile is generated by a finite volume solver, using shock capturing techniques to generate an axisymmetric flow field. Local boundary-layer profiles are extracted from this flow field and hypersonic Mack-modes are superimposed for cone-walls with and without a porous surface used as a passive transition-reduction device. Special care is taken of curvature effects of the wall on the mode development over smooth and porous walls.

\section{INTRODUCTION}

A typical challenge for reentry vehicles during a flight is the effective control of the hypersonic transition from the laminar to the turbulent state of the boundary layer. In this work, the passive manipulation of the transition is performed by the use of porous surfaces to influence the growth of the second mode, which is an acoustic instability. The second or so-called Mack-mode [1] is the dominant mode for the transition process at hypersonic Mach numbers and the first goal of the transition control in this regime is to prevent or delay the growth of Mackmodes. The ability of a microporous surface to delay the transition, at least in some cases, was demonstrated by Maslov [2] in shock tunnel experiments of the flow over a cone. The effect was found to become stronger as the Knudsen number increased, as modeled by the linear theory within the slip flow regime. Fedorov et al. [3] used a different type of surface and demonstrated that similar effects could be achieved with thin porous coatings with a random microstructure. As shown in former studies, the effect of porous walls with a uniform and nonuniform pore 


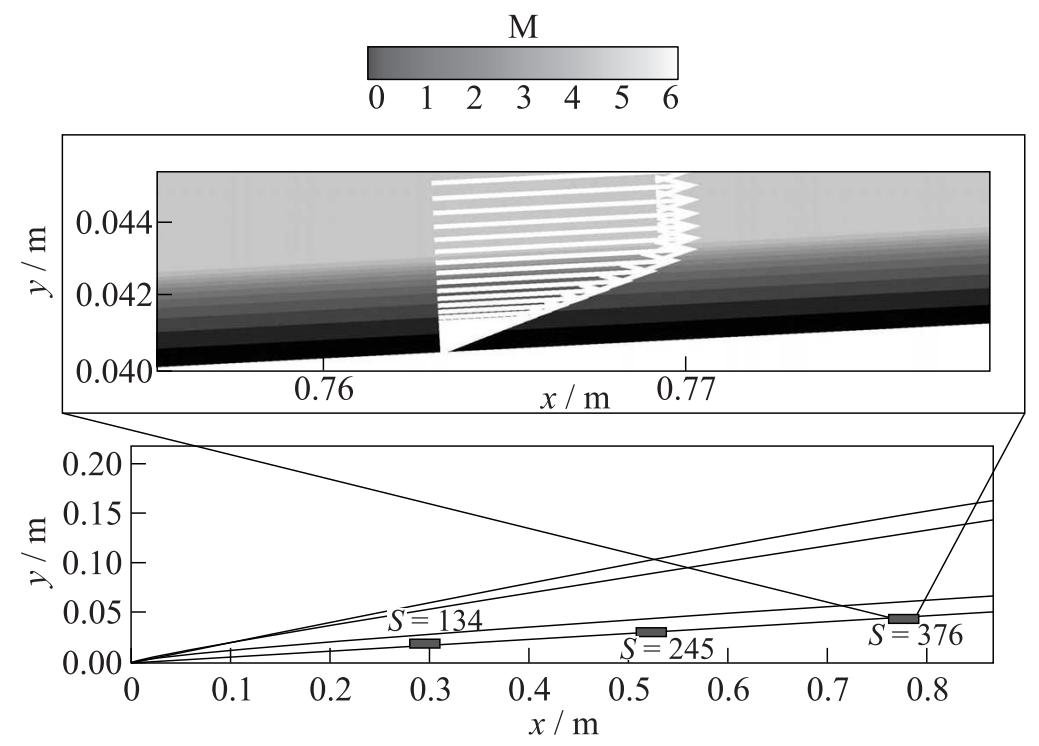

Figure 1 Mach number distribution on the hypersonic cone and extracted boundarylayer profile

distribution and adiabatic as well as cold walls, can be simulated successfully by two- (2D) and three-dimensional (3D) DNS-calculations. These studies are carried out by a DNS of the resolved rather than modeled pores with a 4th order finite difference code, which is well validated for various geometries $[4$, 5]. The resolved pores allow an evaluation of the accuracy of simplified onedimensional models used in investigations based on the linear stability theory and this evaluation is carried out very successfully in the mentioned studies.

For wind tunnel experiments, mainly conical models are used due to the axisymmetric flow field that does not necessitate a special treatment of boundary conditions, but provides a flow defined by only one plane through the axis (Fig. 1).

For these wind tunnel conditions, different studies with porous walls are carried out by LST with absorptive boundary conditions implemented. For DNS calculations, it is possible to adapt these boundary conditions for Navier-Stokes solvers, but up to now, these configurations were never simulated by DNS with resolved pores. Interesting questions with respect to these configurations is the influence of the wall curvature on the Mack-modes in contrast to the local conical boundary layer used for DNS calculations over a flat plate. The LST simulations suggest a minor influence of the curvature terms on the growth rates near the transition, but a verification of this statement by DNS is a necessity for future studies. 
Such a conical flow field over a 3 degree blunt cone is investigated in the present paper with respect to a hypersonic stability analysis of the boundarylayer flow at Mach 6 with and without porous walls by comparing local DNS and LST data. The original boundary-layer profile is calculated by a second order finite volume solver, using shock capturing techniques to generate an axisymmetric flow field (see Fig. 1). Special care is taken of curvature effects of the wall on the mode development on smooth and porous walls.

\section{NUMERICAL METHODS}

Two simulation techniques are used in this work. Direct numerical simulations are used to provide complete solutions for the flow over and in the pores, while a linear stability code was used as a cheaper prediction method. A temporal formulation, with periodic boundary conditions in the streamwise direction, was chosen for comparison since this allows an efficient concentration of grid points to resolve multiple pores within one wavelength of the instability. Indeed, the simulations would not be feasible if the spatially-developing approach was taken, due to the large computational cost.

\section{Direct Numerical Simulation}

The equations to be solved are the compressible Navier-Stokes equations for the flow of a perfect gas with the density $\rho$, the velocity components $u_{i}$, the pressure $p$, and the internal energy $e$, written in conservation law form as

$$
\begin{gathered}
\frac{\partial \rho}{\partial t}+\frac{\partial \rho u_{j}}{\partial x_{j}}=0 ; \\
\frac{\partial \rho u_{i}}{\partial t}+\frac{\partial \rho u_{i} u_{j}}{\partial x_{j}}+\frac{\partial p}{\partial x_{i}}=\frac{\partial \tau_{i j}}{\partial x_{j}}+f_{i} ; \\
\frac{\partial \rho E}{\partial t}+\frac{\partial(\rho E+p) u_{i}}{\partial x_{i}}=-\frac{\partial q_{i}}{\partial x_{i}}+\frac{\partial u_{i} \tau_{i j}}{\partial x_{j}}+g
\end{gathered}
$$

where $E=e+u_{i} u_{i} / 2$. Forcing terms $f_{i}$ and $g$ are included in the right hand side of the equations so that a specified parallel base flow $\bar{\rho}(y), \bar{u}_{i}(y), \bar{E}(y)$ is time independent. In practice, these terms are evaluated numerically within the code by computing and storing the initial residual. The base flow and the forcing terms are zero within the pores. This approach means that the laminar base flow is maintained exactly for smooth walls, whereas for porous walls the flow is able to adjust to a local solution of the governing equations. This allows the formation of a small slip velocity when an average streamwise velocity is computed over the wall plane. 
The equations are closed with the perfect gas law together with the constitutive relations for $q_{i}$ and $\tau_{i j}$ [5]. The normalization of the flow quantities is carried out for all spacial coordinates by the displacement thickness $\delta^{*}$, for the pressure by $P_{\infty}$ and for the velocities by

$$
V_{\text {ref }}=\sqrt{\frac{P_{\infty}}{V_{\infty}}}
$$

at the boundary-layer edge. The reference time-scale is consequently chosen by

$$
t_{\mathrm{ref}}=\frac{\delta^{*}}{V_{\mathrm{ref}}} .
$$

The base flow is obtained from a separate solution of the cone-geometry by the second-order finite-volume version of the FLOWer code, including shock treatment. A base flow inside the $\mathrm{H} 2 \mathrm{~K}$ wind tunnel in Cologne at Mach number $\mathrm{M}=6$, Reynolds number $\operatorname{Re}=28,738$, Prandtl number $\operatorname{Pr}=0.72$, the ratio of specific heats $\gamma=1.4$, and an adiabatic wall boundary condition is considered. The viscosity $\mu$ is prescribed by Sutherland's law with the constant of $110.4 \mathrm{~K}$ and the reference temperature of $64.63 \mathrm{~K}$, leading to the wall temperature of $434 \mathrm{~K}$. The same Sutherland coefficients are used for the thermal conductivity $\kappa$. This boundary-layer profile is extracted inside the bow-shock before being interpolated onto the DNS-grid by a cubic spline method. While former DNS of porous surfaces have calculated instabilities in flat-plate boundary layers [5], for the first time, a profile at real wind-tunnel conditions from the tested conegeometry is chosen.

To study the curvature effect on the Mack-mode development in this profile, it was placed artificially at different streamwise locations of the cone surface near the beginning, middle and end of the porous surface-part. Consequently, only one parameter is changed and the growth rates are kept comparable, even though the real profiles at these positions differ significantly. The profile is taken from the end of the porous layer at the surface co-ordinate $S^{*}=761 \mathrm{~mm}$ on the wind tunnel model, and at the displacement thickness of $\delta^{*}=2.069$, resulting in a nondimensional coordinate of $S=376$. The other positions are $S=245$ and 134 , all nondimensionalized by the same $\delta^{*}$.

The DNS-calculations in this paper are made with a high-order variant of the DLR FLOWer code. The basic FLOWer code solves the compressible Reynoldsaveraged Navier-Stokes equations on block-structured grids with second-order finite volume techniques and cell-centred or cell vertex variables. The high-order version used in the present work [4] uses fourth-order central differencing based on standard compact finite differences in a cell-centred formulation, together with high-order compact filters that are applied at the end of each time step. Options are available for a skew-symmetric splitting the convective terms and for sponge-zone boundary conditions to reduce reflections. For the present work, a 


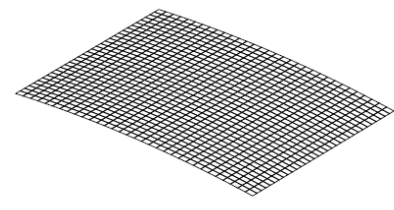

(a)

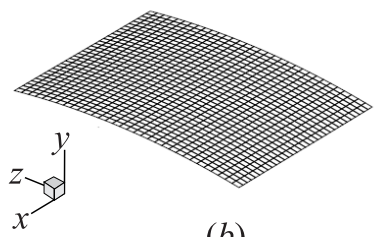

(b)

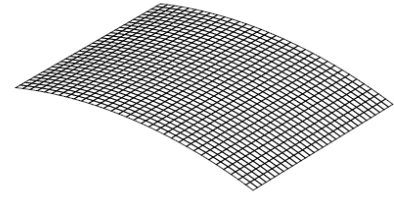

(c)

Figure 2 Surface grids at different positions on the cone: (a) $S=376$; (b) 245; and (c) $S=134$

6th order filter and the standard conservative form of the Euler terms are used. The time advancement is by a three-step second-order Runge-Kutta method [6]. For the present work, the calculations are limited to rectangular pores so that grids match at the interface, removing interpolation as a possible source of error are limited.

A grid stretching function is used in all cases that places more points near $y=0$. Including cases with pores the function is given by

$$
y=L_{y} \frac{\sinh (c \eta)}{\sinh c}
$$

where $\eta_{m}<\eta<1, c$ and $\eta_{m}$ are chosen iteratively near the target $c$ so that $y\left(\eta_{m}\right)=-d$, with $d$ being the pore depth, and an integer number of grid cells within the pore. For the conical meshes, these 2D grids are tilted and turned around the cone-axis with 32 spanwise cells to generate local parts of the smooth-cone surface as shown in Fig. 2. The increasing curvature at the decreasing streamwise coordinate is evident. Two different spanwise extents of $0.5 \delta^{*}$ and $4.0 \delta^{*}$, which denote the arc-length at the respective position, are chosen to demonstrate the influence of this dimension. This is especially important for the simulation of pores where the spanwise extent is with $0.32 \delta^{*}$ even smaller to limit the large computational costs for more than four pore-rows.

An overview of the conical grid with pores of 0.4834 depth is shown in Fig. 3 at $S=134$. The curvature in the pores is particularly visible at the first plane with four pores. The calculations use periodic boundary conditions in the $x$ and $z$ directions. The use of a periodic boundary condition in $x$ corresponds to the temporal formulation of the linear stability theory. This approach is supported by [7] and [5] where it was found that spatial and temporal results could be interchanged to a good accuracy using the Gaster transformation, at least for Blasius-profiles. It has to be pointed out that the Gaster transformation as a first-order approximation can reduce comparability for the chosen conditions due to significant deviations of the boundary layer from the Blasius approach. A no-slip adiabatic wall is applied at $y=0$ and on surfaces within the pores. At the outer boundary, the characteristic conditions are used. 


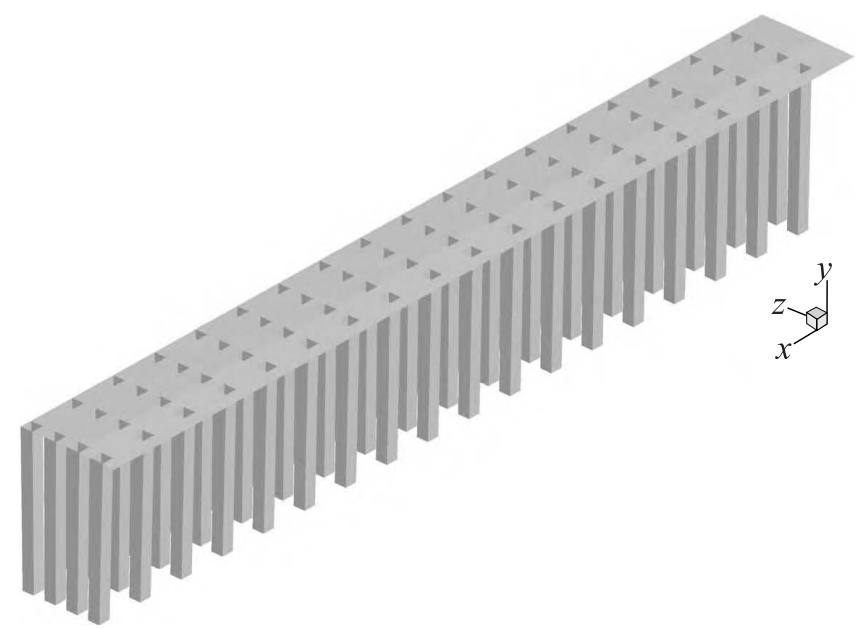

Figure 3 Surface geometry of the calculated porous layer at $S=134 ; 18 \times 4$ pores in stream- or spanwise direction

\section{LINEAR STABILITY THEORY}

The NOLOT code, NOnLocal Transition analysis code [8], was developed in cooperation between DLR and FOI and can be used for local as well as nonlocal analyses. In this work the local linear spatial approach is used, which is a subset of the nonlocal stability equations. The equations are derived from the conservation equations of the mass, the momentum, and the energy, which govern the flow of a viscous, compressible, ideal gas, formulated in primitive variables. All flow and material quantities are decomposed into a steady laminar base flow $\bar{q}$ and an unsteady disturbance flow $\tilde{q}$

$$
q(x, y, z, t)=\bar{q}(x, y)+\tilde{q}(x, y, z, t)
$$

The laminar base-flow $\bar{q}$ is calculated by the finite-volume version of the DLR FLOWer code. As described above, this code solves the compressible Reynoldsaveraged Navier-Stokes equations for a perfect gas flow on block-structured grids, using second order finite volume techniques and cell-centered or cell vertex variables.

The disturbance $\tilde{q}$ is represented as a harmonic wave

$$
\tilde{q}(x, y, z, t)=\hat{q}(x, y) \exp [i(\alpha x+\beta z-\omega t)]
$$

with the complex-valued amplitude function $\hat{q}$. In the following, the hat over a variable denotes the amplitude function. Since NOLOT is a spatial code the 
wavenumbers $\alpha$ and $\beta$ are the complex quantities and the frequency $\omega$ is the real value. The parameter $\alpha_{i}$ is the growth rate, which is the quantity of primary interest. The boundary conditions in NOLOT for a smooth wall (at $y=0)$ are:

$$
\hat{u}_{w}, \hat{v}_{w}, \hat{w}_{w}, \hat{T}_{w}=0 .
$$

The NOLOT code is validated by several test cases against published results, including DNS, PSE (parabolized stability equations), multiple scales methods and LST. A good summary of the validation is given by Hein et al. [8]. For the treatment of porous surfaces, additional boundary conditions are implemented. The verification of this porous boundary condition against the Southsampton LST code and DNS is given in $[9,10]$. The validation of LST against experiments on the smooth and porous cone in the H2K of the DLR Cologne can be found in [11] where a good agreement between experiments and the present approach is shown. The boundary conditions for porous walls are taken from [2], a complete derivation can be found in these references. The conditions are given by

$$
\hat{u}_{w}, \hat{v}_{w}=0 ; \quad \hat{w}_{w}=A \hat{p}_{w} ; \quad \hat{T}_{w}=B \hat{p}_{w}
$$

where a subscript $w$ denotes the value at the wall. Fedorov et al. have shown that the thermal admittance $B$ has a marginal effect [12]. It is consequently neglected in the present work.

The pores are taken to be circular with the radius $r$, and all variables are normalized with respect to free stream values, with a reference length given by the displacement thickness of the boundary layer. The pores of different geometries, namely, 2D grooves and circular or quadratic pores are kept comparable by he hydraulic diameter, defined as $d_{h}=4 A / C$ where $A$ is the pore area and $C$ is the circumference. The hydraulic diameter was suggested by Fedorov et al. in 2003 as a method for comparing pores with different structure and is widely used in various studies [9].

\section{RESULTS}

\subsection{Curvature Influence on Mack-Modes in a Smooth-Cone Boundary Layer}

For all following simulations, the aforementioned boundary-layer profile at windtunnel conditions is chosen. From linear stability theory, the wavenumber of the peak-growth-rate is chosen [5] which is $\omega_{i}=2.8575$. It has to be mentioned that for all following calculations, only qualitative comparisons between DNS and LST are possible, since the NOLOT code includes pressure and velocity gradients for the stability calculations, while these influences are omitted in the 


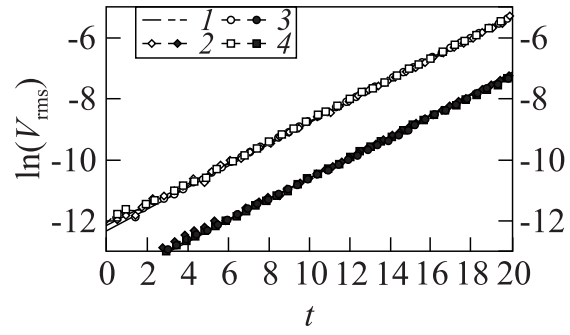

(a)

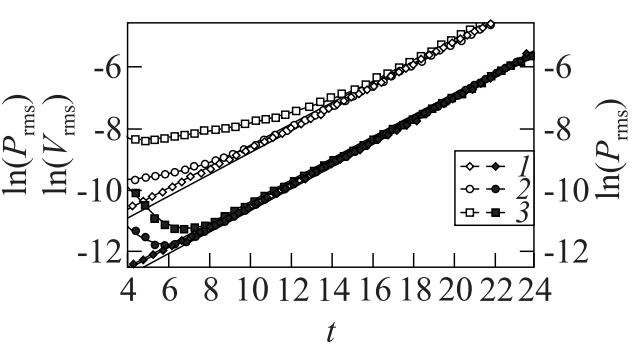

(b)

Figure 4 Mack-mode growth in time on a smooth cone: $(a)$ for different spanwise grid-extents $\left(1-\right.$ NOLOT-LST; $2-S=376, L_{z}=0.5 ; 3-S=376, L_{z}=4.0$; and 4 - flat, $\left.L_{z}=4.0\right)$ and (b) for different streamwise positions on the cone $(1-$ $S=376 ; 2-245$; and $3-S=134$ ). Linear fits of the growth rates are added by straight lines without symbols. Empty symbols refer to $\ln \left(V_{\mathrm{rms}}\right)$ and filled symbols refer to $\ln \left(P_{\mathrm{rms}}\right)$

DNS. Nevertheless, for the smooth cone, comparisons are shown to demonstrate the reasonable agreement of the two approaches.

The DNS is set up on a grid, using 32 points in the streamwise $x$ direction and 320 points in the wall-normal direction $y$, in which the grid was stretched with a coefficient $c=3.5$ in (1). The DNS is initialized with an artificial disturbance at $t=0$ and the eigenmode is allowed to develop from this. The initial disturbance is given by

$$
v=0.0001 \exp \left[-4(y-0.8)^{2}\right] \sin \left(\frac{2 \pi x}{L_{x}}\right)
$$

with $y$ as the wall-normal co-ordinate on the cone and all other variables initialized to the base flow condition.

For different spanwise grid-extents, Fig. $4 a$ shows the development of the natural logarithm of the root mean square of the $v$ and $p$ perturbations (integrated over the $y$ direction) as the simulation proceeds. By $t=4$, the unstable mode has emerged and grows strongly over the remainder of the simulation. For this stage of linear growth, linear fits through the respective data points are carried out and added to the diagrams by using the same line patterns as denoted in the legend but without the symbols that mark original DNS data (see, e.g., Fig. 4). An exception are the LST-simulations in Fig. 4a, for which the straight black lines are also added in the legend. Though at later time-stages the modes for $L_{z}=4.0$ spanwise instabilities can be expected in the Mack-mode development, the growth rates $\omega_{i}$ in the linear regime between $t=4$ and 20 are nearly independent from the spanwise extent. In addition the curvature effect is shown to be minor, since the calculation for a flat plate is also very similar in the growth rate, as well as the LST calculation with NOLOT. The DNS results 
differ from each other by less than $0.3 \%$ and from the LST-simulation by less than $2.5 \%$. At different streamwise positions, the situation differs (Fig. $4 b$ ). The relaxation from the initial perturbation to the linear mode-growth takes significantly longer for larger curvatures and the growth rate differs by around $2.6 \%$ between the largest slope at $S=134$ and the smallest at $S=376$. Generally the influence of the curvature is minor for the investigated case, since even the largest nondimensional curvature $\kappa$, defined by $\kappa=\delta^{*} / r_{\text {local }}$, is in a range of 0.14 , which is still a small value.

\subsection{Grid Influence for Porous Surfaces}

In this section, the investigations of grid-influences in the DNS-calculations with pores are presented. For the experimental setup, 80-micron circular pores at the porosity of 0.125 are distributed over the cone. For the given condition, 18 pores/wave-length result from this prescription at a hydraulic diameter of $80 \mu \mathrm{m}$ or an equivalent groove diameter of $40 \mu \mathrm{m}$. Quadratic pores of $80 \mu \mathrm{m}$ in diameter are distributed in two or four spanwise rows for the $3 \mathrm{D}$ computations.

Contours of the wall-normal velocity are shown in Fig. 5 for $2 \mathrm{D}$ and $3 \mathrm{D}$ pore geometries after the development of instabilities. As a first step, 2D simulations at $S=376$ with spanwise grooves are compared at different cell-numbers inside. 6,8 , and 12 cells are distributed through the grooves and the results are very close (Fig. $6 a$ ) at deviations in the range of $1.3 \%$. Consequently, 6 cells inside

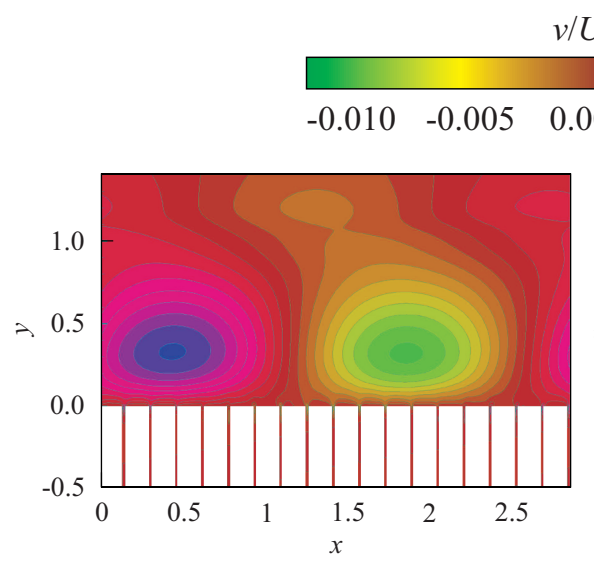

(a)

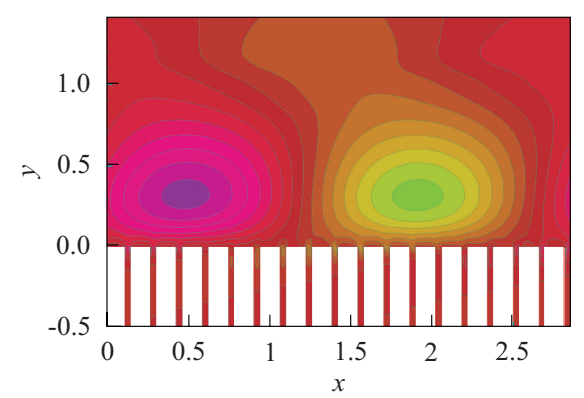

(b)

Figure 5 The $v$-contours of Mack-modes over 2D grooves $(a)$ and 3D quadratic pores $(b)$. 


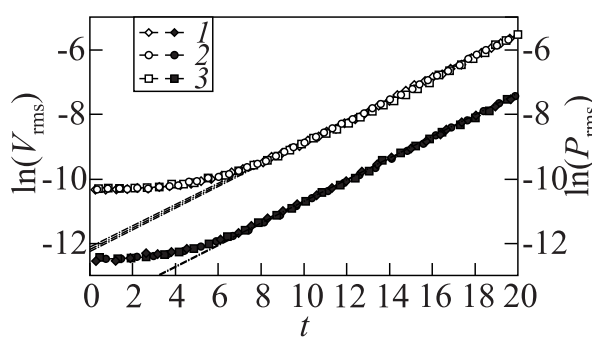

(a)

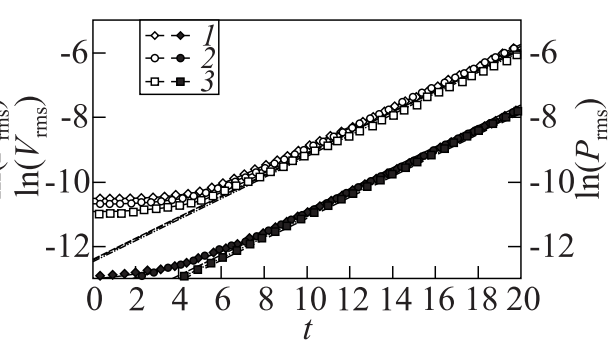

(b)

Figure 6 Mack-mode growth in time on a porous cone: $(a)$ for $2 \mathrm{D}$ influence of different cell-numbers through the grooves $(1-6$ cells per pore; $2-8$; and $3-$ 12 cells per pore) and $(b)$ for $3 \mathrm{D}$ influence of different spanwise rows of pores $(1-$ $18 \times 2$ pores, $S=376 ; 2-18 \times 4$ pores, $S=376$; and $3-18 \times 4$ pores, flat). Linear fits of the growth rates are added by straight lines without symbols. Empty symbols refer to $\ln \left(V_{\text {rms }}\right)$ and filled symbols refer to $\ln \left(P_{\text {rms }}\right)$

the pores are shown to be sufficient for the present study. The influence of the pore-number in spanwise direction is significantly stronger (Fig. 6b). The growth rates for 2-rows of pores and a calculation without any curvature deviate only by $1.8 \%$, since the spanwise extent for two pores is only $1 / 9$ of the wavelength. The growth-rate difference between two and four spanwise rows is with $3.8 \%$ more than doubled, which can be expected for the doubled spanwise extent. For this reason, all grids with quadratic pores are generated with four spanwise rows.

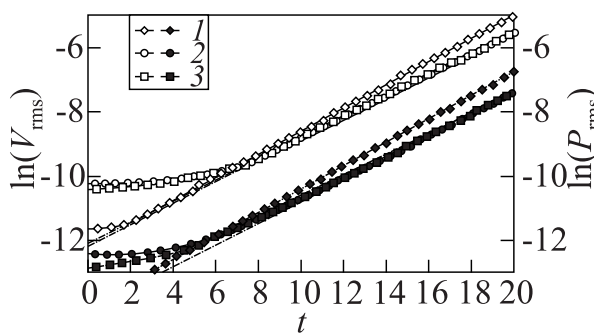

(a)

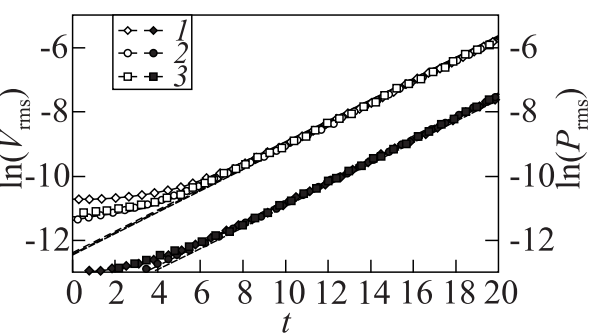

(b)

Figure 7 Mack-mode growth in time on a porous cone: $(a)$ for comparison of grooves and pores with equal hydraulic diameter $(1-$ no pores, $2 \mathrm{D} ; 2-18$ pores, $2 \mathrm{D}$; and $3-18 \times 4$ pores, $S=376$ ) and (b) for quadratic pores at different streamwise positions $(1-S=376 ; 2-245$; and $3-S=134)$. Linear fits of the growth rates are added by straight lines without symbols. Empty symbols refer to $\ln \left(V_{\mathrm{rms}}\right)$ and filled symbols refer to $\ln \left(P_{\text {rms }}\right)$ 


\subsection{Curvature Effects on the Porous Cone}

For comparison of different pore geometries, the hydraulic diameter is introduced in former sections. The influence of this simple quantity for comparison of complex pore geometries is investigated in former studies [10], and is very useful for deep pores. In Fig. $7 a$, a comparison of growth rates from $2 \mathrm{D}$ grooves and quadratic pores with equal hydraulic diameter shows a good agreement. The curvature effect on the growth rate over a porous coating is demonstrated in Fig. $7 b$ where the same tendency as for the smooth cone is visible. Again, the largest slope is found at $S=134$ and the smallest at $S=376$. Even the deviation in the range of $2.3 \%$ is similar to the value for a smooth cone.

\section{CONCLUDING REMARKS}

Within this study, a second mode stability analysis is performed for a boundarylayer flow at Mach 6 over a smooth cone and porous coatings at different positions along the cone surface. For this investigation, the boundary-layer profile is generated by a finite volume computation of the axisymmetric cone inside the DLR$\mathrm{H} 2 \mathrm{~K}$ wind tunnel. Only one representative boundary-layer profile is extracted and the growth of second-mode instabilities in the base-flow profile is simulated by DNS for the same profile at varying spanwise curvatures, corresponding to the respective position. As usual, the maximum-amplified wavelength is chosen for the Mack-mode and the DNS is designed as a temporal approach. It could be shown that the curvature of the cone surface on the growth rate of the instabilities is minor for this case from a wind-tunnel-model geometry. A grid convergence study of different cell numbers inside $2 \mathrm{D}$ grooves is carried out and different numbers of spanwise rows for quadratic pores are investigated. The hydraulic diameter has shown to be an adequate similarity parameter for different pore shapes, even for this axisymmetric flow-field. By this study, the former investigations on Mack-modes in boundary layers neglecting curvature effects for the stability calculation are justified at least for the investigated model geometry.

\section{REFERENCES}

1. Mack, L. M. 1984. Boundary layer linear stability theory. AGARD Special Course on Stability and Transition of Laminar Flow.

2. Maslov, A. A. 2003. Experimental and theoretical studies of hypersonic laminar flow control using ultrasonically absorptive coatings (UAC). Report ISTC 2172-2001.

3. Fedorov, A., A. Shiplyuk, A. Maslov, E. Burov, and N. Malmuth. 2003. Stabilization of a hypersonic boundary layer using an ultrasonically absoptive coating. J. Fluid Mech. 479:99-124. 
4. Enk, S. 2008. A fourth order finite difference method for large eddy simulation at a flat plate. 16 DGLR-Fach-Symposium der STAB. Aachen, Germany.

5. Sandham, N.D., and H. Lüdeke. 2009. A numerical study of Mach 6 boundary layer stabilisation by means of a porous surface. AIAA J. 47(9):2243-52.

6. Kroll, N., and R. K. Jain. 1987. Solution of two-dimensional Euler equations experience with a finite volume code. DFVLR Forschungsbericht 87-41.

7. Bres, G. A., T. Colonius, and A. V. Fedorov. 2008. Stability of temporally-evolving supersonic boundary layers over micro-cavities for ultrasonic absorptive coatings. AIAA Paper No. 2008-4337.

8. Hein, S., F. P. Bertolotti, M. Simen, A. Hanifi, and D. Henningson. 1994. Linear nonlocal instability analysis - the linear NOLOT code. DLR-IB 223-94 A56.

9. Wartemann, V., H. Lüdeke, and N.D. Sandham. 2009. Stability analysis of hypersonic boundary layer flow over microporous surfaces. 16th AIAA/DLR/DGLR Space Planes and Hypersonic Systems and Technologies Conference (International). Bremen, Germany.

10. Wartemann, V., and H. Lüdeke. 2010. Investigation of slip boundary conditions of hypersonic flow over microporous surfaces. ECCOMAS 2010. Lisbon, Portugal.

11. Wartemann, V., and H. Lüdeke. 2011. Stability analyses and validation of a porous surface boundary condition by hypersonic experiments on a cone model. 7th Aerothermodynamics Symposium. Brugge, Belgium.

12. Fedorov, A. V., N. D. Malmuth, A. Rasheed, and H. G. Hornung. 2001. Stabilization of hypersonic boundary layers by porous coatings. AIAA J. 39(4):605-10. 